REPORTE DE CASO

\title{
COLITIS AMEBIANA FULMINANTE CON DESENLACE FATAL EN UN PACIENTE CON PROBABLE INFECCIÓN POR SARS-COV-2
}

\author{
José Somocurcio@11,2a, Tula Ayquipa@1,3,b, Vanessa Pineda@11,4, \\ 1 Hospital Nacional Edgardo Rebagliati Martins, Lima, Perú. \\ 2 Universidad Nacional Mayor de San Marcos, Lima, Perú. \\ 3 Universidad Ricardo Palma, Lima, Perú. \\ ${ }^{4}$ Universidad Peruana Cayetano Heredia, Lima, Perú. \\ a Médico anatomopatólogo, magíster en Medicina; ${ }^{b}$ médico residente de Anatomía Patológica; ${ }^{\circ}$ médico residente de \\ Radiología.
}

\section{RESUMEN}

Se reporta el caso de un varón de 62 años quien presentó tos y dolor abdominal por dos semanas, con hallazgos tomográficos pulmonares compatibles con probable infección por SARS-CoV-2, que recibió tempranamente corticoterapia a dosis altas y de manera ambulatoria. El paciente presentó evolución tórpida, fue hospitalizado y falleció en el posoperatorio inmediato por un cuadro de abdomen agudo quirúrgico. El estudio anatomopatológico mostró estructuras parasitarias con características compatibles con amebas, como causa de una colitis aguda fulminante complicada, con perforación múltiple y peritonitis aguda. La colitis aguda fulminante por amebiasis intestinal en el contexto de un paciente con probable COVID-19 no había sido reportada en el Perú. Resulta importante resaltar la presentación fulminante con desenlace fatal de esta infección parasitaria prevalente en nuestro medio, en el contexto del uso de corticoides ante una probable infección pulmonar por COVID-19.

Palabras clave: Amebiasis; COVID-19; SARS-CoV-2; Corticoides; Colitis Amebiana; Parásitos (fuente: DeCS BIREME).

\section{FULMINANT AMEBIC COLITIS WITH FATAL OUTCOME IN A PATIENT WITH PROBABLE SARS-COV-2 INFECTION}

\section{ABSTRACT}

We report the case of a 62-year-old male with cough and abdominal pain for two weeks, associated with pulmonary tomographic findings compatible with probable infection by SARS-CoV-2, and who received high doses of early corticotherapy as an outpatient. The patient showed clinical deterioration, was hospitalized and died in the immediate postoperative period due to acute surgical abdomen. The anatomopathological study showed parasitic structures with characteristics compatible with amebae, which was pointed to as the cause of a complicated acute fulminant colitis, with multiple perforations and acute peritonitis. Acute fulminant colitis due to intestinal amebiasis in the context of a patient with probable COVID-19 had not been previously reported in Peru. It is important to highlight the fulminant presentation with fatal outcome of this prevalent parasitic infection, in the context of corticosteroids use in a probable SARS-CoV-2 pulmonary infection.

Citar como: Somocurcio S, Ayquipa T, Pineda V. Colitis amebiana fulminante con desenlace fatal en un paciente con probable infección por SARS-CoV-2 Rev Peru Med Exp Salud Publica. 2021;38(3):458-62. doi: https://doi. org/10.17843/rpmesp.2021.383.8050.

\section{Correspondencia:}

José René Somocurcio Peralta; Av. Juan De Aliaga 426, Dpto. 901, Magdalena Del Mar, Lima, Perú; jsomocurcio@yahoo.es

Recibido: 08/05/2021 Aprobado: 29/09/2021 En línea: 31/09/2021
Keywords: Amebiasis; COVID-19; SARS-CoV-2; Corticosteroides; Amebic Colitis; Parasites (source: MeSH NLM).

\section{INTRODUCCIÓN}

La amebiasis intestinal es una enfermedad causada por el parásito Entamoeba histolytica, frecuente en todo el mundo, pero de mayor prevalencia en países en vías de desarrollo. La mayoría de los pacientes infectados son asintomáticos, sin embargo, existe un grupo reducido que desarrolla formas invasivas como la colitis amebiana, siendo la colitis aguda fulminante la forma más severa de presentación ${ }^{(1)}$. Esta se asocia a una alta morbilidad y a una mortalidad de hasta $99 \%$. Los factores de riesgo para su desarrollo son el uso de corticoides, estado de malnutrición, inmunosupresión y embarazo ${ }^{(2)}$. En el contexto de la actual pandemia por la COVID-19, el uso de corticoides se ha incrementado, pues ha demostrado que disminuye la mortalidad en pacientes hospitalizados con requerimiento de oxígeno ${ }^{(3)}$, lo que podría estar relacionado a cuadros severos de infecciones preexistentes desencadenando parasitosis fulminantes. 


\section{REPORTE DEL CASO}

Se presenta el caso de un varón de 62 años, procedente del distrito de San Juan de Lurigancho de la ciudad de Lima, con antecedentes de hipertensión arterial, psoriasis e hiperplasia benigna prostática en tratamiento regular. El tiempo de enfermedad fue de dos semanas e inició con tos y dolor abdominal, siendo considerado como caso sospechoso de COVID-19 en una primera atención médica, por lo cual recibió antibióticos y altas dosis de corticoides. Una semana después, el paciente presentó deposiciones líquidas con moco y sangre, por lo que acudió al servicio de emergencia de un hospital de referencia nacional.
El paciente ingresó con funciones vitales estables. En el examen físico se hallaron crepitantes pulmonares bibasales, distensión abdominal, dolor a la palpación profunda y superficial del abdomen, predominantemente en hipocondrio derecho y ruidos hidroaéreos presentes.

En los resultados de laboratorio se halló leucocitosis de 22,770 con $8 \%$ de abastonados, hemoglobina de $8,8 \mathrm{mg} / \mathrm{dL}$ y proteína $\mathrm{C}$ reactiva (PCR) de 30,11 . El perfil bioquímico y la gasometría se encontraban dentro de los rangos normales. Se le realizó la prueba de antígeno para COVID-19, con resultado negativo; sin embargo, la tomografía de tórax mostró la presencia de opacidades en vidrio esmerilado de distribución central y periférica, confluentes y bilaterales, con compromiso de ápices, zonas medias y bases, y algunos focos
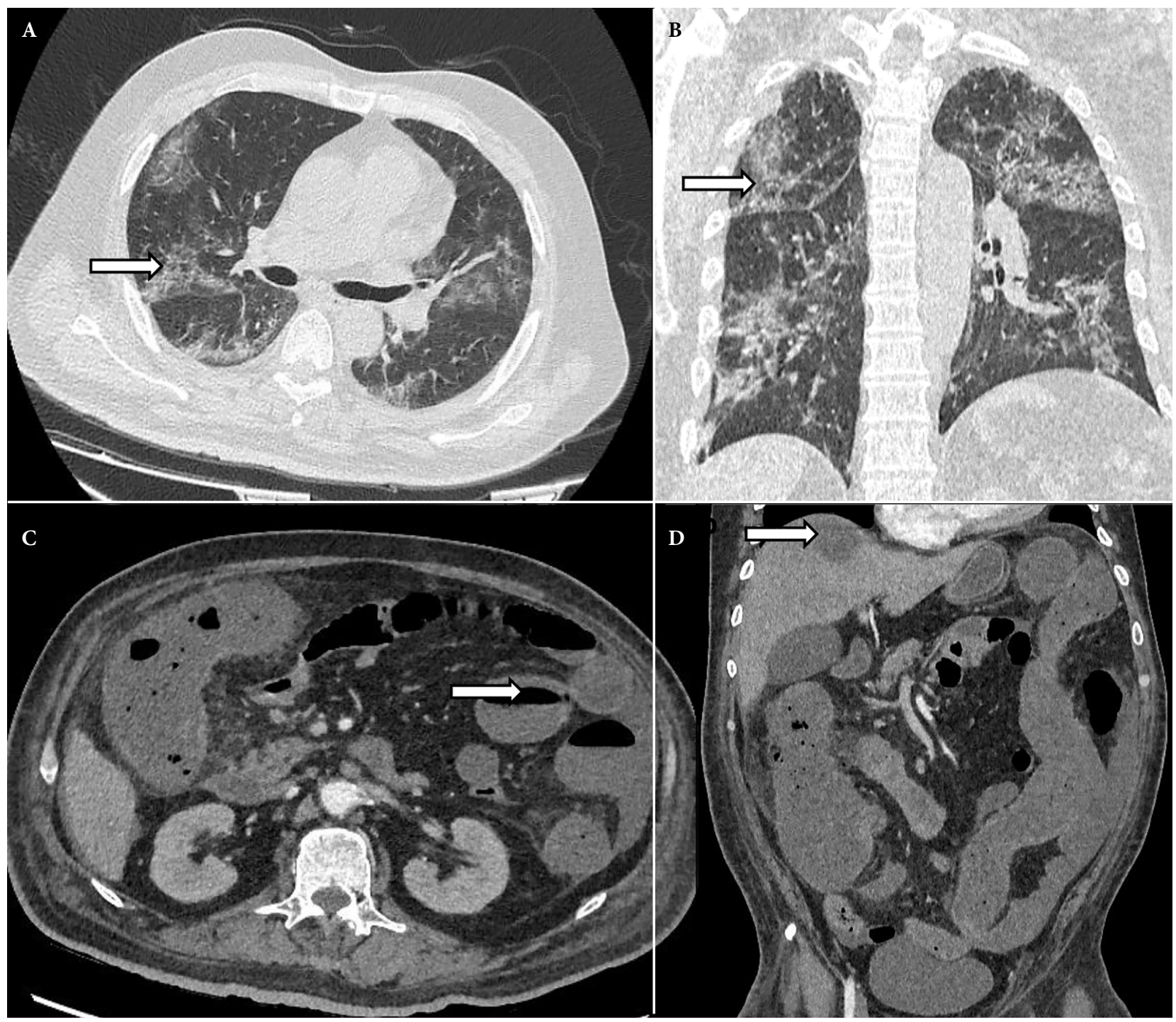

Figura 1. Tomografía de tórax y abdomen. La vista axial (A) y coronal (B) en ventana pulmonar definen opacidades en vidrio esmerilado de distribución central y periférica, confluentes y bilaterales, que comprometen los ápices, zonas medias y bases; hallazgos típicos de neumonía por COVID-19 (CO-RADS 5) en fase viral, con afectación pulmonar del 50\% (flecha). La vista axial (C) muestra cambios inflamatorios de la pared del marco colónica y niveles hidroaéreos en asas intestinales delgadas (flecha), hallazgos con relación a colitis e íleo adinámico. La reconstrucción en coronal (D) presenta una imagen compatible con absceso hepático en el segmento VIII del hígado (flecha). 
de consolidación en lóbulos inferiores, siendo estos hallazgos considerados típicos de neumonía por COVID-19 (CO-RADS 5) (Figura 1, A-B). La tomografía de abdomen mostró cambios inflamatorios de la pared del marco colónico a predominio del colon ascendente en relación con signos de colitis, y una imagen hipodensa con realce en anillo, compatible con absceso en el segmento VIII del hígado (Figura 1, C-D). Se consideraron los diagnósticos de pancolitis de probable origen infeccioso versus origen isquémico, y la probable infección por SARSCoV-2. Se le indicó manejo expectante con antibioticoterapia (meropenem y vancomicina), así como oxígeno suplementario condicional.

En el día 11 de hospitalización, el paciente presentó incremento del dolor y distensión abdominal, en una nueva tomografía se observaron cambios inflamatorios de la pared colónica y del íleon distal, con signos de perforación en el colon derecho. El paciente ingresó a sala de operaciones; donde le encontraron múltiples perforaciones en el colon derecho e izquierdo, y exudado purulento con afectación del íleon y recto. Se le realizó colectomía total y resección del íleon distal; sin embargo, el paciente falleció en el posoperatorio inmediato.

El resultado de anatomía patológica reportó el compromiso del colon derecho, izquierdo, recto sigmoides e íleon por múltiples lesiones ulceradas, algunas perforadas, de contenido necrótico, de base ancha y con trombosis vascular arteriolar secundaria, con presencia de estructuras parasitarias unicelulares con eritrocitos intracitoplasmáticos compatibles con trofozoítos de Entamoeba histolytica, corroboradas por pruebas complementarias de inmunohistoquímica e histoquímica (Figuras 2, 3 y 4).

\section{DISCUSIÓN}

La amebiasis intestinal es la infección del tracto gastrointestinal causada por el parásito Entamoeba histolytica ${ }^{(4)}$. Este patógeno se transmite por vía fecal-oral y es el responsable de diarreas severas en adultos y niños, siendo la tercera causa de muerte por parasitosis a nivel mundial. ${ }^{(5)}$ Además, un reanálisis reciente del Estudio multicéntrico entérico global (GEMS, por sus siglas en inglés), demostró que esta ameba es uno de los siete patógenos que causan disentería con mayor frecuencia en el mundo ${ }^{(6)}$.

La distribución de la Entamoeba histolytica es cosmopolita y es más frecuente en países en vías de desarrollo, especialmente en zonas con medidas de saneamiento limitadas, sin acceso a agua segura ni desagüe. Utilizando la técnica de reacción en cadena de la polimerasa (PCR) se ha reportado su presencia en el 8,5\% de las muestras de heces analizadas en un estudio de Sudáfrica y en el $13,7 \%$ en la India ${ }^{(1)}$. Se ha descrito una seroprevalencia de hasta $42 \%$ en algunas zonas rurales de México ${ }^{(7)}$. Si bien, en el Perú no se cuenta con información nacional sobre su prevalencia, un estudio realizado en escolares de la costa norte del país determinó la presencia de este parásito en un 4,5\% de las muestras de heces evaluadas ${ }^{(8)}$.

La Entamoeba histolytica es un protozoario del género Entamoeba de la familia Entamoebidae que también incluye a las especies E. dispar, E. moshkovskii, E. bangladesi, E. polecki, E. hartmanni y E. coli ${ }^{(9)}$. Estas especies han sido identificadas en el tracto intestinal del hombre; sin embargo, solo algunas de ellas son consideradas patógenas, siendo la infección por Entamoeba histolytica la más agresiva ${ }^{(9)}$. Se reporta que hasta el $90 \%$ de los pacientes colonizados por este protozoario permanecen asintomáticos, mientras que el 10\% de ellos desarrollan la enfermedad invasiva en la forma de colitis amebiana, absceso hepático y otras manifestaciones fulminantes de menor frecuencia ${ }^{(10,11)}$.

La virulencia in vitro está dada por la capacidad de adherencia, citotoxicidad, actividad proteasa, destrucción de la monocapa, motilidad, fagocitosis y trogocitosis que se ha visto confirmada en resecciones colónicas donde se observa degradación de la mucosa colónica, migración a través de
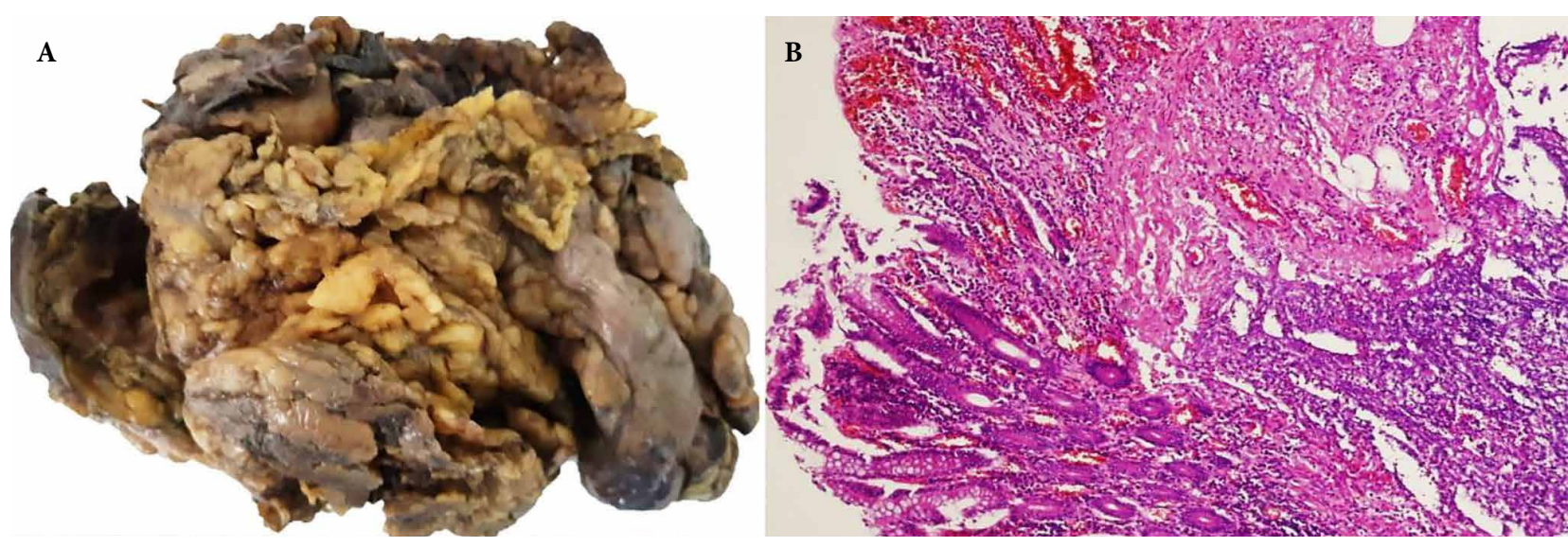

Figura 2. Resección de colon izquierdo. (A) Perforación y peritonitis purulenta. (B) Úlcera necrótica «en botón de camisa». Tinción con hematoxilina y eosina $10 \mathrm{X}$. 


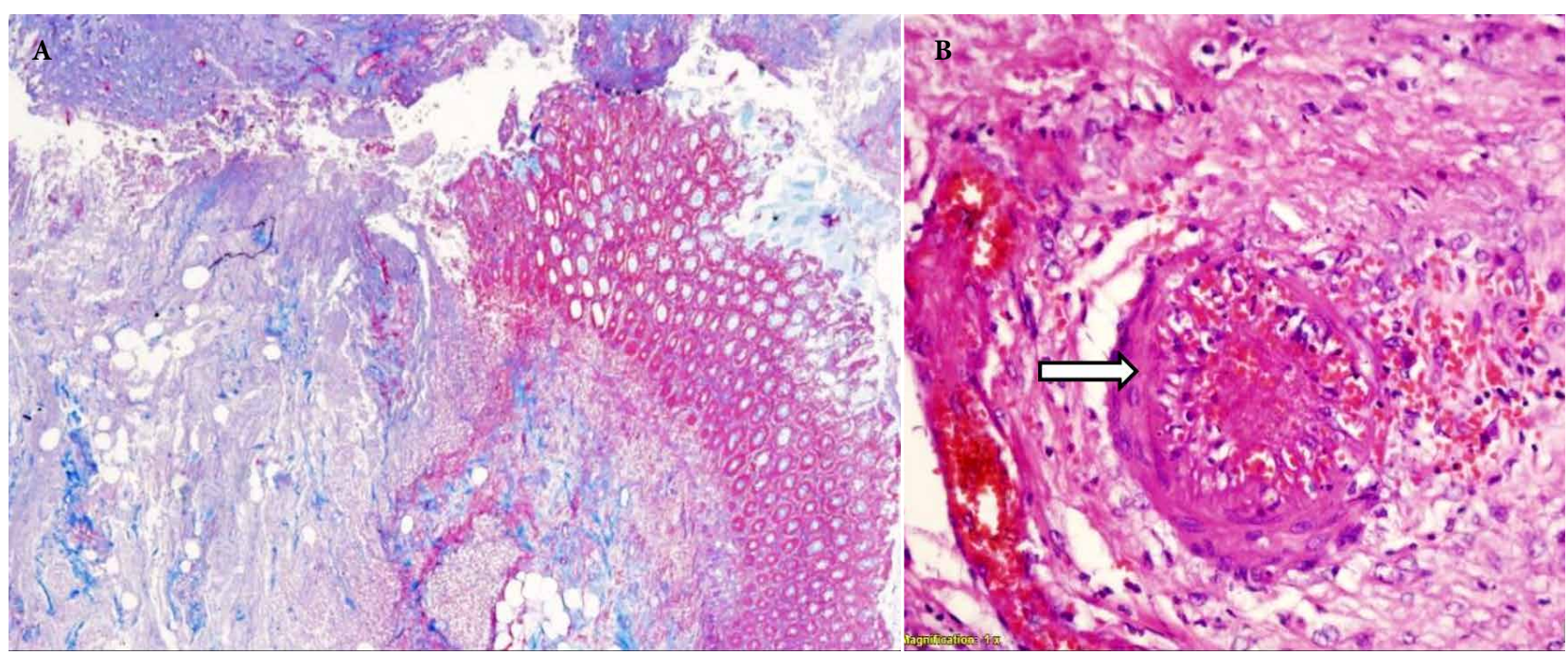

Figura 3. Resección de colon derecho. (A) Extensas úlceras necróticas con afectación de capa muscular. Tinción con tricrómica de Masson 5X. (B) Trombosis vascular (flecha). Tinción con hematoxilina y eosina 40X.

la malla de colágeno, degradación de la matriz extracelular, muerte celular e invasión tisular ${ }^{(5)}$. No obstante, in vivo, en la presentación clínica influyen factores del hospedero y del genotipo ${ }^{(5)}$. Su transmisión es a través de la vía fecal-oral ${ }^{(1)}$. Se ingiere en forma de quiste que se encuentra en alimentos, agua o suelos contaminados ${ }^{(11)}$. Al llegar al íleon, se produce el enquistamiento del parásito el cual adquiere la forma de trofozoito, estadio invasivo que afecta principalmente el intestino grueso, ocasionando cuadros de disentería ${ }^{(11)}$.

Las formas invasivas intestinales pueden evolucionar a perforaciones múltiples que alcanzan una mortalidad del $50 \%$ al $100 \%{ }^{(1)}$. Esta presentación predomina en pacientes inmunosuprimidos ${ }^{(1,12,13)}$, aunque también se ha reportado en pacientes inmunocompetentes ${ }^{(1)}$. Las principales manifestaciones clínicas son dolor abdominal, fiebre y leucocitosis, hallazgos que se encontraron en nuestro paciente.

El diagnóstico se realiza mediante serología o microbiología en heces; aunque, debido al curso fulminante, se define en los estudios histológicos post mortem ${ }^{(1)}$, tal como sucedió en el presente reporte. El tratamiento de elección es el metronidazol endovenoso ${ }^{(1)}$.

La amebiasis extraintestinal se presenta en el $2 \%$ a $3 \%$ de los pacientes y se da por diseminación hematógena a través de la circulación enteroportal mediante la cual llega al hígado y produce abscesos hepáticos amebianos (14). Asimismo, mediante esta vía, a través de la vena cava superior, puede llegar

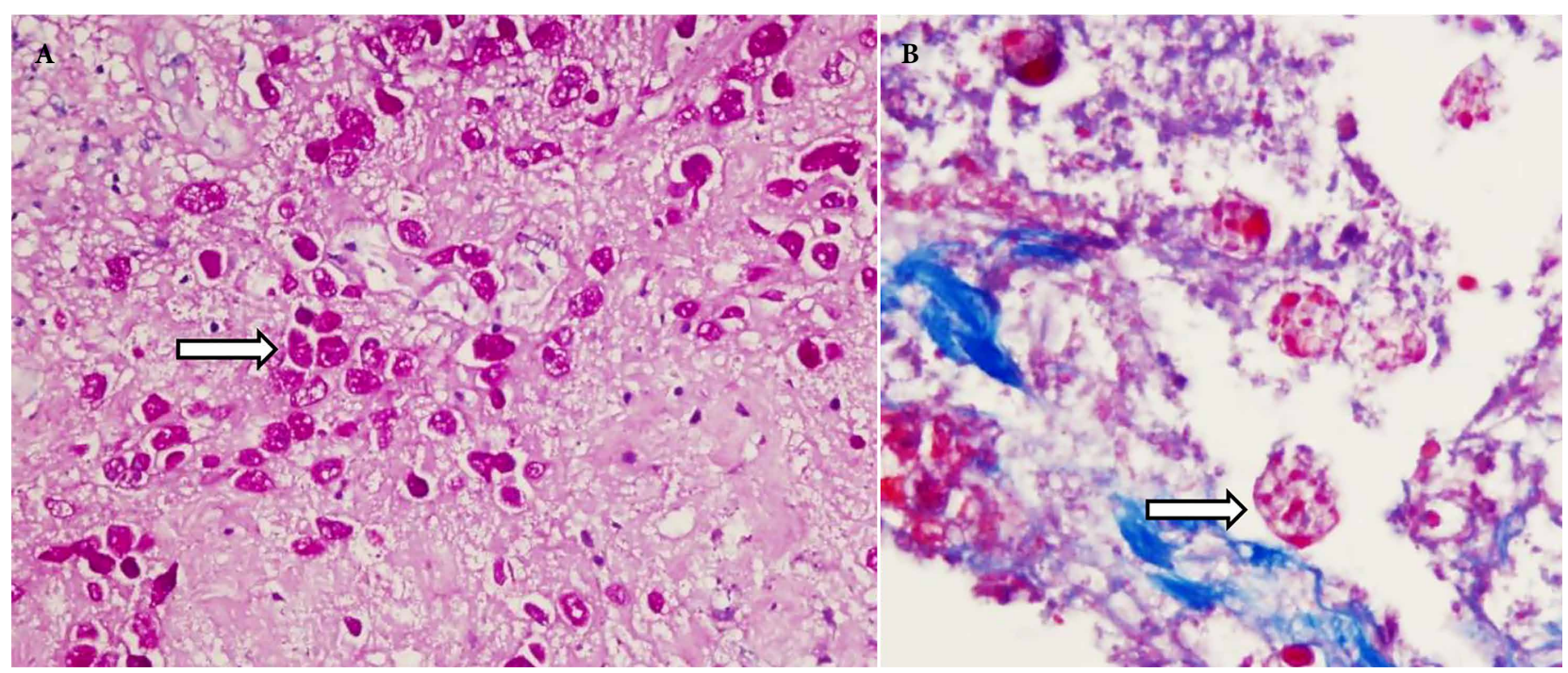

Figura 4. Destrucción tisular. (A) Invasión masiva de trofozoitos de Entamoeba histolitica en el tejido colónico (flecha). Tinción con PAS 40X. (B) Amebas con eritrofagocitosis (flecha). Tinción con tricrómica de Masson 100X. 
al pulmón originando abscesos pulmonares primarios, aunque estos son muy raros ${ }^{(14)}$. Por lo general, la principal causa de afectación pulmonar es por continuidad a la ruptura de un absceso hepático ${ }^{(14)}$. Si bien no se ha descrito una presentación pulmonar difusa primaria, estas características se encuentran en casos de ruptura de abscesos pulmonares con afectación bronquial ${ }^{(14)}$, lo cual no ocurrió en nuestro paciente.

La infección por SARS-CoV-2 concomitante a un cuadro de amebiasis intestinal fulminante no se había descrito previamente. Aunque, en el caso presentado, la infección viral no pudo confirmarse mediante pruebas serológicas ni antigénicas, la descripción tomográfica fue altamente sospechosa de COVID-19 (CORADS-5) y, excluyendo presentaciones amebianas pulmonares similares que expliquen el compromiso pulmonar, se consideró una infección probable por COVID-19 relacionada con un cuadro de colitis amebiana fulminante. Además, nuestro paciente tenía factores de riesgo para desarrollar un cuadro severo de COVID-19 (edad avanzada e hipertensión arterial), por lo que recibió de manera ambulatoria altas dosis de corticoesteroides por al menos ocho días, lo que podría haber ocasionado la presentación amebiana letal que se reporta. Los autores queremos resaltar que es imprescindible considerar la infección por E. histolytica y otros agentes infecciosos preexistentes, como Strongyloides stercoralis o Mycobacterium tuberculosis, antes de la administración de corticoesteroides, debido a su potencial daño, tal como lo señala la revisión sistemática de Shirley et al. en la que se observa que el $25 \%$ de los pacientes con amebiasis intestinal

\section{REFERENCIAS BIBLIOGRÁFICAS}

1. Shirley DT, Farr L, Watanabe K, Moonah S. A Review of the Global Burden, New Diagnostics, and Current Therapeutics for Amebiasis. Open Forum Infect Dis. 2018;5(7):ofy161. doi: 10.1093/ofid/ofy161.

2. Shirley DA, Moonah S. Fulminant Amebic Colitis after Corticosteroid Therapy: A Systematic Review. PLoS Negl Trop Dis. 2016;10(7):e0004879. doi: 10.1371/journal.pntd.0004879.

3. RECOVERY Collaborative Group, Horby P, Lim WS, Emberson JR, Mafham M, Bell JL, et al. Dexamethasone in Hospitalized Patients with Covid-19. N Engl J Med. 2021;384(8):693-704. doi: 10.1056/NEJMoa2021436.

4. Espinosa-Cantellano M, Martínez-Palomo A. Pathogenesis of intestinal amebiasis: from molecules to disease. Clin Microbiol Rev. 2000;13(2):318331. doi: 10.1128/CMR.13.2.318.

5. Marie C, Petri WA Jr. Regulation of virulence of Entamoeba histolytica. Annu Rev Microbiol. 2014;68:493-520. doi: 10.1146/annurev-micro-091313-103550.

6. Liu J, Platts-Mills JA, Juma J, Kabir F, Nkeze J, Okoi C, et al. Use of quantitative molecular diagnostic methods to identify causes of diarrhoea in children: a reanalysis of the GEMS case-control study. Lancet. 2016;388(10051):1291-1301. doi: 10.1016/S0140-6736(16)31529-X.

7. Alvarado-Esquivel C, Hernandez-Tinoco J, Sanchez-Anguiano LF Seroepidemiology of Entamoeba histolytica Infection in General Population in Rural Durango, Mexico. JClin Med Res. 2015;7(6):435-439. doi: 10.14740/jocmr2131w.

8. Vilches-Berríos GN, Rentería-Valle CA, Monteza-Salazar JL, Silva-Díaz H. Coccidiosis y amibiasis intestinal en niños de edad escolar de un dis- que usaron corticoides murieron, a pesar de la cirugía y del uso de metronidazol ${ }^{(2)}$.

Finalmente, debido a que varios estudios ya han descrito el papel de la COVID-19 en la inmunidad ${ }^{(15)}$ y que el tratamiento de casos severos incluye el uso de corticoides ${ }^{(3)}$, instamos el descarte de infecciones parasitarias preexistentes previo al empleo de corticoides a dosis altas y hacemos un llamado a los investigadores para que reporten casos similares, a fin de mejorar la comprensión de la COVID-19 y su tratamiento, en relación con la inmunidad y la aparición de infecciones parasitarias masivas.

Resulta importante la evaluación integral de los pacientes con factores de riesgo para desarrollar cuadros severos de COVID-19 antes de iniciar el tratamiento con corticoides. En nuestro medio, las patologías infecciosas son prevalentes y deben considerarse en el contexto de la pandemia actual para prevenir el desarrollo de presentaciones fulminantes en pacientes tratados con corticoides.

Criterios éticos: El estudio fue evaluado y aprobado por el Comité Institucional de Ética e Investigación del Instituto de Evaluación de Tecnologías en Salud e Investigación (IETSI) de EsSalud. Se obtuvo el consentimiento informado del familiar del paciente para publicar el reporte.

Contribuciones de los autores: Todos los autores participaron en la concepción y diseño del estudio, análisis e interpretación de datos, redacción y revisión crítica del artículo, y aprobación de la versión final.

Financiamiento: El presente trabajo ha sido financiado por los investigadores responsables.

Conflicto de interés: Los investigadores responsables declaran no tener conflictos de interés. trito de Lambayeque, Perú. Rev Med Hered. 29(1):5-10. doi: 10.20453/ rmh.v29i1.3254.

9. Carrero JC, Reyes-López M, Serrano-Luna J, Shibayama M, Unzueta J, León-Sicairos N, et al. Intestinal amoebiasis: 160 years of its first detection and still remains as a health problem in developing countries. Int J Med Microbiol. 2020;310(1):151358. doi: 10.1016/j.ijmm.2019.151358.

10. Taherian M, Samankan S, Cagir B. Amebic Colitis. In: StatPearls [Internet]. Treasure Island (FL): StatPearls Publishing; 2021. Disponible en: https:// www.ncbi.nlm.nih.gov/books/NBK542237/.

11. Wang H, Kanthan R. Multiple colonic and ileal perforations due to unsuspected intestinal amoebiasis-Case report and review. Pathol Res Pract. 2020;216(1):152608. doi: 10.1016/j.prp.2019.152608.

12. Rodríguez-Wong $\mathrm{U}$, Rodríguez-Medina $\mathrm{U}$. Colitis amibiana fulminante con neumatosis cecal. Rev Gastroenterol Mex. 2018;83(4):453-4. doi: 10.1016/j.rgmx.2018.02.007.

13. Briceño-Santana M, Grijalva NMB, Vargas-Guzmán AL, Manterola C, García Méndez N. Colitis amebiana necrosante, presentación de un caso. Rev Chil Infectol. 2020;37(5):599-603. doi: 10.4067/S071610182020000500599 .

14. DíazSantos, G, Panqueba CA, Lara R, Lastra G. Absceso pulmonar amebiano RFS Revista Facultad De Salud. 2011;3(2): 53-57. doi: 10.25054/rfs.v3i2.75.

15. Mehta P, McAuley DF, Brown M, Sanchez E, Tattersall RS, Manson JJ, et al. COVID-19: consider cytokine storm syndromes and immunosuppression. Lancet. 2020;395(10229):1033-4. doi: 10.1016/S0140-6736(20)30628-0. 